\title{
Personality goes a long way: The malleability of opposite-sex physical attractiveness
}

\author{
GARY W. LEWANDOWSKI JR., ${ }^{a}$ ARTHUR ARON, ${ }^{b}$ AND JULIE GEE ${ }^{b}$ \\ ${ }^{a}$ Monmouth University and ${ }^{b}$ State University of New York, Stony Brook
}

\begin{abstract}
This study tested the influence of personality information on judgments of physical attractiveness. Employing a within-subject design, 56 female and 22 male college students rated attractiveness of opposite-sex photos; participated in a distraction task; viewed each photo again, along with personality information (desirable, undesirable, none); and then rated the photos for physical attractiveness, desirability as a friend, and desirability as a dating partner. Personality information produced significant changes in ratings of physical attractiveness for attractive, neutral, and unattractive targets. The pattern of results is consistent with a model in which desirability of the target's personality leads to greater desirability as a friend, leading to greater desirability as a dating partner, leading to the target being judged as more physically attractive.
\end{abstract}

Physical appearance plays a central role in romantic attraction (e.g., Aron, Dutton, Aron, \& Iverson, 1989; Buss, 1989; Li, Bailey, \& Kenrick, 2002). Understanding the influences on romantic attraction is a longstanding theme in the relationship sciences because of its theoretical and practical importance. This importance is largely due to the significance of romantic love in social experience and directing life choices (Aron, Fisher, \& Strong, 2006) and its role in directing mating processes (Buss \& Kenrick, 1998; Fisher, 1998). Physical appearance is also important more generally in person perception; good-looking people are judged more positively and treated more favorably in a wide variety of contexts (Langlois et al., 2000). Physical appearance is a particularly important factor in attraction because it is

Gary W. Lewandowski Jr., Department of Psychology, Monmouth University; Arthur Aron, Department of Psychology, State University of New York at Stony Brook; Julie Gee, Department of Psychology, State University of New York at Stony Brook.

Correspondence should be addressed to Gary W. Lewandowski Jr., Monmouth University, Department of Psychology, West Long Branch, NJ 07764, e-mail: glewando@monmouth.edu. typically the first information we have about a person.

Perceived personal qualities also play an important role in romantic attraction (e.g., Aron et al., 1989; Buss, 1989), some of which (e.g., kindness) appear to be necessities for attraction (Li et al., 2002), particularly as a relationship develops (Levinger, 1994). In the past, researchers have examined physical appearance and personality mainly as independent sources of variance in predicting attraction. Also, when they studied these two characteristics together, participants have usually received both kinds of information simultaneously, rather than in the more typical context in which seeing the person's appearance precedes learning about their personality (cf. Altman \& Taylor, 1973).

The focus of this article is on the potential influence of subsequently learned personality information in modifying initial physical attractiveness judgments, a possibility consistent with recent research on how cognitive processes and expectations modify perception (Eberhardt, Dasgupta, \& Banaszynski, 2003; von Hippel, Sekaquaptewa, \& Vargas, 1995). Specifically, we predict such an effect, offer a model of the mechanisms by which such an 
effect occurs, and present results of an experiment that tests the basic effect and the overall model.

\section{Background}

The "what is beautiful is good" stereotype (Dion, Berscheid, \& Walster, 1972), a classic phenomenon in social psychology, suggests that when forming impressions of others, perceiving a person as good looking leads to positive expectations about personal characteristics. Meta-analyses (Eagly, Ashmore, Makhijani, \& Longo, 1991; Feingold, 1992) support both that this phenomenon functions as a stereotype (e.g., the typical perceived link between appearance and personality is larger than the actual link) and that the effect, though not as strong or general as originally thought, is indeed found reliably across many studies.

Influence of personality and physical attractiveness on general attraction. Research that has examined the potential interaction of physical and nonphysical information on romantic attraction has yielded mixed results. Lampel and Anderson (1968) had 40 female participants rate the dating desirability of males after viewing a photograph accompanied by personality traits (i.e., temporally stable and consistent aspects of one's personality) such as friendly and messy. Both physical and personality trait information that influenced desire to date interacted such that traits were more influential (in the positive direction) with attractive photographs. Keisling and Gynther (1993) had 122 male participants rate the general attractiveness of females after viewing pictures of attractive, average, and unattractive females, each accompanied by information related to masculine or feminine traits. Participants rated physically attractive females and those with more feminine traits as more attractive, with an interaction such that the personality effect held in the average and unattractive physical attractiveness conditions but not in the attractive condition. Finally, Kleck and Rubenstein (1975) examined 48 males' attraction to a female confederate who was or was not physically attractive (manipulated through makeup and hair style) and who had similar or dissimilar attitudes. The confederate's appearance was the only significant influence on attraction. Thus, there has been some research, with mixed results, on the joint role of physical appearance and perceived personality on general attraction.

Influence of personality on perception of appearance. This research does not reveal the potential influence that personality information may have on perceptions of appearance. Consistent with the halo effect (Nisbett \& Wilson, 1977), global positive or negative evaluations of a person can influence ratings of specific features such that raters view identical features on the same person differently depending on context in which they are presented. For example, the general perception of physical size can be influenced by context, such that identical female faces are seen as smaller when grouped among male faces compared to other female faces (Stapel \& Koomen, 1997). This can also occur for more specific physical features such as a person's height. Wilson (1968) found that estimates of height were positively correlated with academic status such that raters viewed those with higher status as taller.

It is also possible that information about a person can influence the perception of specific facial features. Relevant to this possibility, Hassin and Trope (2000) had 120 twelfthgrade students view pictures of a face, along with a paragraph describing the person pictured as either mean or kind, and then rate several facial features. Compared to targets described as mean, raters perceived kind targets as having shorter ears, rounder chins, fuller and wider faces, and higher attractiveness. Similarly, a study that examined perceptions of crime witnesses, found that participant's with negative perception of the witness (i.e., witnesses that did not help) also perceived the witness's facial features differently compared to witnesses that did offer help to the victim (Veenvliet \& Paunonen, 2005). Specifically, raters perceived the negatively viewed witnesses as having longer noses, thinner lips, chins that were more narrow and square, and faces that were more pale and narrow compared to positively viewed witnesses. 
If personality information can influence perception of individual facial features, it seems plausible that it can also influence perceptions of physical attractiveness.

Influence of personality on perception of physical attractiveness. We were able to locate a few studies relevant to the possibility that personality information can specifically affect judgments of a target's physical attractiveness. Hassebrauck (1986), focusing on attitude similarity, had 59 male and female participants rate the physical attractiveness of a photograph depicting a male of average attractiveness presented along with the target's attitudes (manipulated to be similar or dissimilar to the participant's responses in an earlier questionnaire). Participants rated the target male as more physically attractive when he supposedly had similar attitudes. Another study focused directly on personal information, examining a "what-is-good-is-beautiful stereotype" (Gross \& Crofton, 1977). In this study, 125 male and female participants rated female targets' physical attractiveness based on a photograph and a profile containing personality and physical information. The photographs were of a target who was physically attractive, average, or unattractive (based on previous ratings). Researchers manipulated favorability of personality descriptions by altering information related to grade point average, value to a group, and several trait-related adjective pairs (e.g., selfishgenerous). Attractiveness of the photograph and the favorability of the personality description significantly influenced targets' ratings of physical attractiveness (there was no interaction).

More recently, Paunonen (2006) presented male and female participants with a brief personality description that characterized the target on three dimensions (honesty, intelligence, independence). After reading the description, participants made personality inferences, and then rated a set of same-sex pictures of average attractiveness. This helped ensure that participants based perceptions of physical characteristics on the personality information. Results indicate that honesty was positively associated with perceptions of greater overall physical fitness and health. Participants rated faces of targets in this condition as kinder, more femi- nine, and more attractive. Liking for the target mediated these effects. This study is important because it suggests a potential mechanism for the perception of physical appearance. Due to the absence of a pretest, it is unclear from this design if perceptions of physical attractiveness change over time.

These studies support the notion that personal information can affect ratings of physical attractiveness. For present purposes, these important studies were limited in three key respects. First, treatment of target gender across studies was not ideal for assessing attractiveness relevant to heterosexual romantic attraction (targets were of the same sex in the Hassebrauck, 1986, and the Gross \& Crofton, 1977, study; targets were of same or opposite sex in the Paunonen, 1996, study), while observers were both male and female, and reported results only for combined ratings by both sexes. Second, researchers presented physical appearance and trait information simultaneously or without establishing a baseline rating of target physical attractiveness. This makes an analysis concerning how judgments of physical attractiveness may change over time problematic. Third, and most important, this previous research did not examine intervening mechanisms of physical attractiveness change.

\section{Potential mechanisms: A model}

Interaction appearance theory examines potential general mechanisms for physical attractiveness change in the context of communication processes (Albada, Knapp, \& Theune, 2002). According to this theory, people hold expectations about potential partners' physical attractiveness and communication ability, such that when they meet a person who does not quite meet expectations in terms of physical attractiveness, the nature of the social interaction is a key determinant of the ultimate attraction. Specifically, if the interaction is positive enough, the perceiver will increase the original perception of physical attractiveness in order to be congruent with the positive social interaction. This model suggests that a primary intervening mechanism for physical attractiveness change is the social interaction 
process. Due to the complex nature of social interactions, it is difficult to isolate the true cause of the change in physical attractiveness perception. That is, it is possible that continued interaction produced increases in perceived attractiveness due to a mere exposure effect (Moreland \& Beach, 1992). Alternately, it is possible that changes from social interaction are the result of verbal or nonverbal communication that conveys information other than about the target's personality (such as reciprocal liking). So while it is likely that social interactions are a major influence on changes in perceived physical attractiveness in naturalistic conditions, it is difficult to pinpoint the specific elements of these interactions that are most responsible.

In an attempt to accomplish this, we propose the following motivational model. Perceiving a person as having a desirable personality makes the person more suitable in general as a close relationship partner of any kind (friend, family member, romantic partner). Thus, one who is more suitable as a relationship partner in general has the potential to be seen as more suitable as a dating partner. In contrast, those who are not viewed as a suitable relationship partner in general would be less likely to be considered as a potential dating partner. Finally, because physical appearance is especially important in a dating partner, it would be beneficial to see a person who is otherwise suitable as a dating partner as also more physically attractive. That is, we are likely to see a person who is desirable as a dating partner as physically attractive because desirable dating partners are expected to be, and desired to be, physically attractive. Further, we propose that this model should operate in the ecologically typical (at least in Western contexts) situation in which one is first exposed to physical appearance and then later learns about the personal characteristics. Figure 1 summarizes the model.

This model seems especially likely in the context of evolutionary theory. According to this theory, a mating partner is desirable to the extent they possess desirable characteristics that could be passed on to offspring, or characteristics that would promote the offspring's survival (Buss \& Barnes, 1986). Previous research in this theory has established the impor- tance of physical appearance (Perrett, Burt, \& Penton-Voak, 1998), personality traits (Buss \& Barnes, 1986), the relative importance of each (Buss, 1989), and how when forced to choose between the two, people will make trade-offs that differentially weight one aspect over the other (Li et al., 2002). The present study considers the additive nature of physical and personality information such that one does not come at the expense of the other. Thus, the effects of information on perceptions of physical attractiveness could be expected to be parallel such that positive information results in increased attraction and negative information in decreased attraction.

Personality information may facilitate attraction because it helps indicate the extent to which a person would be a high-quality relationship partner. Knowing that a person is kind and compassionate is important for forming quality friendships. The more involved nature of romantic relationships makes the potential partner's appropriateness as a friend even more important. In fact, research shows that friendship is an important part of romantic relationships. When researchers asked couples married over 15 years why their marriage lasted, couples cited first that their partner was their best friend and, second, that they liked their partner as a person (Lauer \& Lauer, 1985). Another study reports that $44 \%$ of premarital young adults describe their romantic partner as their best friend (Hendrick \& Hendrick, 1993).

From an evolutionary perspective, there is an advantage in long-term mating for how the partner will treat the self and for how the partner will treat one's offspring. Specifically, a partner who will be a good friend is also likely to be a good parent to potential offspring. In addition, for women, a male partner with an unfriendly and disagreeable personality is likely to have difficulty forming alliances, resulting in lower status and a decreased ability to provide for their offspring (Jensen-Campbell, Graziano, \& West, 1995).

In short-term relationships, a good personality should also be desirable and advantageous. In fact, a partner with a bad personality would be a disadvantage for males because one's offspring would be raised by a lower quality parent and a disadvantage for females because her 


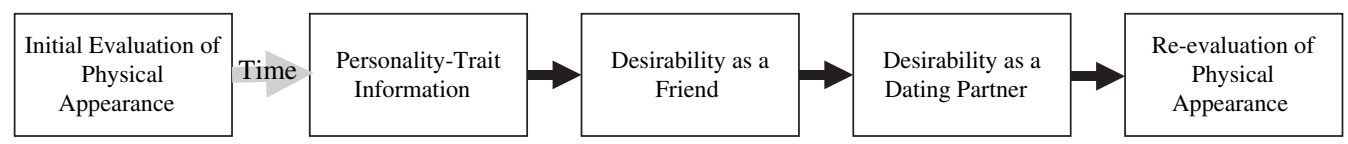

Figure 1. Motivational model for the influence of personality on judgments of physical attractiveness.

offspring might develop those bad personality traits and be less likely to be chosen as a longterm mate in the future. Even if the effect were less in short-term mating, it would be in terms of magnitude, not direction of the effect. Further, since men may be more likely to follow short-term strategies, one might expect bigger effects of personality on perceived attraction for women due to the greater potential costs of having a partner with a negative personality. This is consistent with general findings showing that nonphysical characteristics such as personality have a greater influence on attraction to a romantic partner for women (e.g., Aron et al., 1989; Kniffin \& Wilson, 2004).

\section{The present research}

Employing a within-subject design, women and men rated photos of opposite-sex individuals; participated in a distraction task, viewed the photos again combined with trait information manipulated to be either desirable or undesirable or no information, and then rated the photos again for physical attractiveness as well as for desirability as a friend and as a dating partner. This procedure permitted us to test whether there would be a systematic effect of personal information on change in ratings of physical attractiveness and to assess the fit of the data to our hypothesized fourstage model.

\section{Method}

\section{Participants}

Participants were 78 undergraduates (56 women and 22 men) from a large public and moderately competitive university in the suburban Northeastern United States who took part in the study as part of an introductory psychology research participation requirement. Ages ranged from 18 to $44(M=22.5)$. Of those indicating ethnicity, $28.2 \%$ were Caucasian, $14.1 \%$ were Asian American, $7.7 \%$ were African American, 7.7\% were Hispanic American, and $5.1 \%$ were other. Most were seniors $(54.0 \%)$ and currently in a romantic relationship (64.1\%). As has been typical of attraction experiments in which the focus has been, as it is here, primarily on theory testing and internal validity, participants were from a convenience sample. We did not, however, select participants based on their interest in this particular study, so the findings reported here may be indicative of what would be found in replications where the participants are undergraduate students taking introductory psychology at major North American universities.

\section{Materials}

Personality trait lists. We selected an initial set of traits from previous lists (Anderson, 1968; Kirby \& Gardner, 1972). Pilot testing using male and female raters $(N=34)$ identified the 36 most and least desirable traits to create 12 desirable and 12 undesirable word triads. Example desirable trait triads are honest, humorous, and mature and intelligent, polite, and helpful. Example undesirable trait triads are abusive, offensive, and unstable, and cruel, unfair, and rude. Pilot participants rated the desirable (vs. undesirable) triads as significantly more desirable, $t(34)=247.82, p<$ .001 . Male and female versions used the same trait triads.

Picture stimuli. We selected photos of Caucasian females and males from graduates in a previous year's yearbook from a school in 
another part of the state. We cropped photos within a standard-sized circle with a black background such that only the head of the target was visible and overlaid the circle on a white background. In pilot testing, 72 male and female students from the same university as the present study rated 154 pictures. From this group, everyone rated the female photographs, while approximately half of the 72 students rated the male photographs. Based on the ratings, we used the top, middle, and bottom 12 pictures. These ratings of the resulting stimulus sets were significantly different in the expected direction; for female photos, $F(2$, $69)=73.10, p<.001$; for male photos, $F(2$, $33)=107.74, p<.001$.

\section{Design and procedure}

After giving informed consent, each participant sat alone in a room at a computer with a 17-in. monitor, was read brief instructions about operating the computer, and then the experimenter set up the appropriate protocol using SuperLab 2.0 stimulus presentation software (Cedrus, 1999). All participants viewed pictures of opposite-sex targets; within each gender, we created three versions, counterbalancing so that each level of picture attractiveness was paired with each level of trait information equally over participants. We assigned participants to version via random assignment. The study consisted of four parts: pretest, distraction task, manipulation, and posttest.

Pretest. During pretest, participants viewed 36 pictures representing three levels of picture attractiveness (attractive, average, and unattractive). We counterbalanced the order of picture presentation. Following each picture, the computer prompted participants to "rate the physical attractiveness of the person in the picture" on a scale from 1 (extremely unattractive) to 10 (extremely attractive), using the number keys on the computer. These pretest ratings assessed initial (i.e., prior to exposure to personality information) evaluations of physical attractiveness and also provided a manipulation check on photo attractiveness levels.
Distraction task. Following the pretest, participants engaged in a complex math distraction task to minimize the possibility that initial ratings of photos would be recalled. The task consisted of, first, counting down from a large number (e.g., 9,748) by $7 \mathrm{~s}$; then, after $2 \mathrm{~min}$, the computer instructed participants to use the number they were at and count forward by $13 \mathrm{~s}$; this continued for an additional $2 \mathrm{~min}$.

Manipulation. The manipulation started with 1 of 24 sets of trait triads representing three levels of information (desirable, no information, undesirable). The no-information condition determined if ratings of pictures would change in subsequent ratings without the presence of additional information. After the traits appeared, the computer prompted participants to "rate the attractiveness of the trait information" on a scale from 1 (extremely unattractive) to 10 (extremely attractive). If the computer displayed "no information," participants responded with a 5 . These ratings permitted a check on the personality trait desirability manipulation. Following the rating of the personality information, a photo appeared (one of those presented in the pretest) that ostensibly accompanied the trait information that remained on the screen. This pairing resulted in nine possible combinations of picture (attractive, average, and unattractive) and information (desirable, no information, undesirable). We created pairings of pictures with type of information resulting in three versions of the order (this ensured that each picture and information combination appeared once).

Posttest. Immediately after viewing the picture with the trait ratings, participants rated the physical attractiveness (using the same scale as the pretest). Two additional items asked "How much would you like to date this person?" and "How much would you like to be friends with this person?" Both were rated on a 1 (not at all) to 10 (very much) scale. This question order helps rule out recency effects. That is, one would expect attractiveness ratings to regress increasingly toward the mean over time, and thus, we would expect smaller 
effect sizes as additional ratings are made. Thus, testing all participants in the reverse order of our hypothesized order of effect sizes, we are both working against our hypothesis and providing maximum power for testing this most difficult condition. Additionally, making the question order exactly opposite of the causal order of the hypothesized model, we hoped to minimize alternative explanations to the predicted results. ${ }^{1}$

\section{Results}

\section{Manipulation checks}

Both manipulations were successful. In a oneway univariate analysis of variance (ANOVA), ratings of the trait triads were significantly different in the expected pattern across conditions of desirable trait information $(M=8.00, S D=$ $1.50)$, no information $(M=5.06, S D=0.72)$, and undesirable trait information $(M=1.87$, $S D=1.47), F(2,148)=372.52, p<.001$, partial $\eta^{2}=.91$. Similarly, pretest physical attractiveness ratings were significantly different in the expected pattern across conditions of attractive $(M=6.05, S D=1.38)$, average $(M$ $=4.48, S D=1.20)$, and unattractive $(M=$ $2.95, S D=1.07)$ photos, $F(2,308)=323.33$, $p<.001$, partial $\eta^{2}=.81$.

\section{Change in physical attractiveness ratings}

We conducted a repeated measures univariate ANOVA with the difference between pre- and post physical attractiveness ratings as the

1. A counterbalanced order, in principle, would have had the virtue of permitting a direct test of order effects. Given the likely expectation of regression to the mean for subsequent ratings over time, we would be likely to have found an order interaction with even stronger results for our hypothesis in the other orders. Then, to rule out order effects as an explanation for the overall result, it would be necessary to show that we obtain the results even in the order opposite to our prediction (the one we used in the actual study). We would then be faced with having to test this effect with only one sixth the number of trials available, greatly reducing our statistical power. Further, we are not aware of any reasonable alternative explanations to our predicted results that would be explained by our chosen order. dependent variable. ${ }^{2}$ Attractiveness of picture and desirability of trait information were the independent variables. (We included version in the original analysis as a between-subjects factor. It was not significant in any of the analyses and was thus dropped.) Cell and marginal means are shown in Table 1. As predicted, there was a clear overall main effect of information, in which attractiveness increased from pretest to posttest for desirable trait information $(M=.57)$, stayed about the same for no information $(M=.09)$, and decreased for undesirable trait information $(M=-.92)$, $F(2,154)=73.44, p<.001$, partial $\eta^{2}=.49$. The interaction with picture attractiveness condition was not significant $(F=1.89)$ and the trait desirability effect was significant across each picture attractiveness condition considered separately. There was also a small but significant main effect for picture condition,

2. It is not possible to conduct ANOVAs in a repeated measures design using standard methods. Thus, we also tested whether the pattern reported from the standard ANOVA approach (ANOVA uses, in effect, "gain scores") would also hold for regressed change, which some authorities consider more appropriate. To test for regressed change, we conducted parallel analyses using multilevel modeling carried out with the hierarchical linear modeling (HLM) program (Bryk \& Raudenbush, 1992, version 6.02). Our Level 1 dependent variable was posttest physical attractiveness ratings; we included pretest physical attractiveness ratings as a Level 1 covariate. The two manipulated conditions, also Level 1, were coded as continuous $(-1,0,1)$ and we included their interaction (product term) in the analyses. In every case, results significant in the repeated measures ANOVAs using change scores were also significant in the HLM analyses (and at much more stringent levels). For example, for the main effect of information on physical attractiveness, the coefficient was $.75(p<.001)$. That is, increasing desirability of information by one unit (i.e., undesirable to no information or no information to desirable) predicted an increase of .75 in ratings of physical attractiveness, after controlling for pretest attractiveness. Following recommendations of the American Psychological Association Statistical Task Force (Wilkinson, 1999), we have attempted throughout to apply the most straightforward and familiar methods (in this case, ANOVA) in reporting results in the main text. To be complete, we also report briefly in footnotes the results using HLM, a method that is perhaps less familiar but that may be technically more accurate in some cases. Note that where the same analysis is carried out using both more conventional methods and HLM, results in every single case are entirely consistent (unambiguously significant and in the same direction for both). Also, as in the example in this footnote, in some cases the HLM approach permitted tests that could not be done directly with more familiar methods. 
Table 1. Change in physical attractiveness ratings from pretest to posttest by trait information and picture attractiveness

\begin{tabular}{lcccc}
\hline \hline & \multicolumn{3}{c}{ Information } & \\
\cline { 2 - 4 } Picture & Desirable & No information & Undesirable & Picture mean \\
\hline Attractive & 0.49 & -0.23 & -0.98 & -0.24 \\
Neutral & 0.60 & 0.12 & -1.01 & -0.10 \\
Unattractive & 0.61 & 0.36 & -0.77 & -0.07 \\
Information mean & 0.57 & 0.09 & -0.92 & -0.09 \\
\hline \hline
\end{tabular}

Note. $N$ for each cell $=78$. As predicted, there was a clear overall main effect of information, $F(2,154)=73.44, p<$ .001 , partial $\eta^{2}=.49$. The interaction with picture attractiveness condition was not significant $(F=1.89)$ and the information effect was significant within each picture attractiveness condition considered separately. There was also a small but significant main effect for picture condition, $F(2,154)=4.66, p<.05$, partial $\eta^{2}=.06$.

$F(2,154)=4.66, p<.05$, partial $\eta^{2}=.06$. As shown in Table 1, physical attractiveness decreased slightly from pretest to posttest for attractive pictures but stayed about the same for neutral and unattractive pictures.

\section{Gender differences}

We repeated the above analysis but included gender as a between-subjects factor. The trait information main effect remained clearly significant, $F(2,152)=49.48, p<.001$, partial $\eta^{2}=.39$. There was a small but significant interaction of information condition with gender such that the effect was stronger for women than men, $F(2,152)=4.40, p<.05$, partial $\eta^{2}=.06$. As shown in Figure 2, this was due mainly to women showing a greater difference than men for undesirable to no information, the Gender $\times$ Information interaction for the curvilinear contrast, $F(1,76)=$ $4.92, p<.05$. As can also be seen in Figure 2, the overall effect was quite similar and clearly robust for both genders. Indeed, analyses showed a significant effect for information condition for each gender considered separately: for women, $F(2,110)=67.86, p<$ .001 , partial $\eta^{2}=.55$; for men, $F(2,42)=$ $10.98, p<.001$, partial $\eta^{2}=.34$.

\section{Relationship status}

We also ran a set of univariate ANOVAs that included relationship status as an additional between-subjects factor. Importantly, there were no significant or near-significant effects involving information condition. That is, relationship status did not qualify the entire pattern of results for information effects (including interactions with gender) in any way. We also found one significant effect involving picture condition (but, again, not involving information condition): We identified a significant interaction among picture condition, gender, and relationship status, $F(2,292)=3.58, p<.05$, partial $\eta^{2}=$ .047 . Females who were not in a relationship, relative to those in a relationship, showed a decrease in attraction to the attractive pictures and an increase in attraction to the unattractive pictures; males not in a relationship, compared to those in a relationship, showed the exact opposite pattern.

\section{Four-stage model, differences in effect size}

We tested the four-stage model shown in Figure 1 in two ways. First, we tested the implication of the model that the effect of the information condition should be strongest for the hypothesized most causally proximal variable (friendship desirability), next for the next most proximal (dating desirability), and smallest for the least proximal effect (physical attractiveness). (The effect was significant for each of the three dependent variables considered separately; all $p \mathrm{~s}<.001$.) A $3 \times 3 \times 3$ (information, picture, dependent variable of 


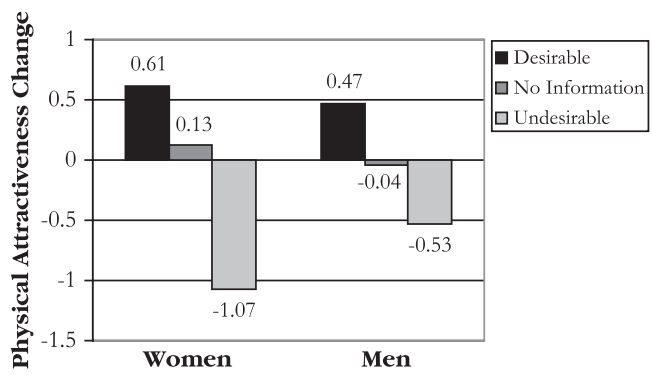

Figure 2. Gender differences in change in physical attractiveness ratings as a function of information desirability.

Note. $N=78$. Information Condition $\times$ Gender interaction, $F(2,152)=4.40, p<.05$, partial $\eta^{2}=.06$. The information main effect was significant and substantial for each gender considered separately, for women, $F(2,110)=67.86, p<.001$, partial $\eta^{2}=.55$; for men, $F(2,42)=10.98$, $p<.001$, partial $\eta^{2}=.34$.

friendship, dating, physical) univariate repeated measures ANOVA showed the predicted interaction of information by dependent variable, $F(4,152)=113.89, p<.001$, partial $\eta^{2}=.63$.

Figure 3 displays the predicted pattern of effect sizes. Comparisons between the effects of each pair of dependent variables were also significant. First, the effect of information on desirability as friend (.80) was significantly greater than on desirability as a dating partner $(.72), F(2,154)=73.68, p<.001$. We calculated this based on the Friendship Desirability versus Dating Desirability $\times$ Information interaction in a Friendship Desirability versus Dating Desirability $\times$ Information $\times$ Picture

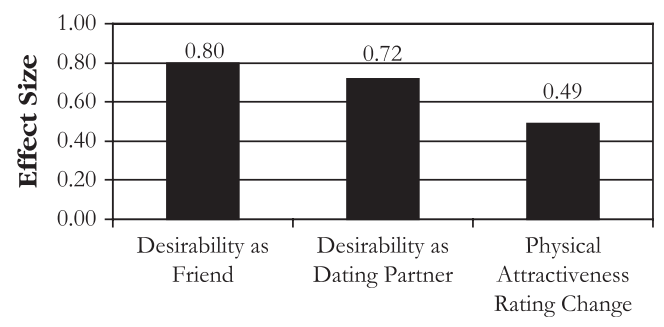

Figure 3. Information condition effect sizes (partial $\eta^{2}$ ) across dependent variables.

Note. $N=78$. Difference in effect size, $F(4$, $152)=113.89, p<.001$, partial $\eta^{2}=.63$. (Each pairwise comparison was also significant at $p<.001)$. repeated measures ANOVA. Similarly, the effect of information on desirability as a dating partner (.72) was significantly greater than on physical attractiveness rating change (.49), $F(2,152)=167.27, p<.001$. There were some cases of interactions with picture attractiveness level or gender, but none of these qualified the basic result, which held for each attractiveness level and each gender considered separately.

Effect sizes for the three dependent variables in the HLM analyses, as predicted from information condition (and including picture condition and the Picture $\times$ Information interaction), also followed this pattern. Controlling for pretest physical attractiveness ratings in all analyses, the coefficients were 2.40 for friendship desirability, 1.57 for dating desirability, and 0.75 for physical attractiveness. The same analyses not controlling for pretest physical attractiveness yielded nearly identical results (2.40, 1.58, and 0.76; in all six analyses, in dichotomous comparisons of effects sizes, $p<.001)$. Using HLM, we also computed the coefficients for each step in the conceptual model shown in Figure 1: from personality trait information to desirability as a friend, $b=2.40$; from desirability as a friend to desirability as a dating partner, $b=.71$; and from desirability as a dating partner to change in physical attractiveness, $b=.54$ (all coefficients, $p<.001$ ). 
Four-stage model, mediation tests

Following the procedures spelled out by Judd, Kenny, and McClelland (2001) for applying the standard Baron and Kenny (1986) approach to within-subjects experimental designs, we tested our four-stage hypothesized model examining mediations. ${ }^{3}$ Step 1 in the standard approach tests whether there is an overall effect to be mediated; that is, it tests the effect of initial cause on the effect. This is done in the within-subjects case with a

3. We also conducted all mediation tests in HLM, following the standard Baron and Kenny (1986) logic. In each case, results were entirely consistent with those reported here using the within-subjects $t$ test and ANOVA results. That is, all steps supported significant and "complete" mediation. For example, consider the mediation by dating desirability of the hypothesized friendship desirability effect on physical attractiveness (all controlling for pretest physical attractiveness). (This example is for the one test that cannot be done at all with the within-subjects $t$ test and ANOVA method.) Step 1, the effect of friendship desirability on physical attractiveness was clearly significant (raw effect size $=.35, p<.001$ ); Step 2 , the effect of friendship desirability on dating desirability was clearly significant $(.65, p<.001)$; Step 3 , the effect of dating desirability on physical attractiveness controlling for friendship desirability was clearly significant $(.55, p<.001)$; and Step 4 , the effect of friendship desirability on physical attractiveness controlling for dating desirability was clearly not significant $(-.01)$. We also evaluated all possible alternative mediations that were inconsistent with the models. (There are a limited number because condition, which was manipulated, can only be a cause, not an effect or mediator). In none of the possible alternative mediational models were results consistent with full mediation; that is, in each case, the cause-to-effect coefficient remained strong and significant when including the mediator. Specifically, information condition predicted desirability as a friend even after controlling for desirability as a dating partner $(b=1.28, p<.001)$ and change in attractiveness rating $(b=2.01, p<.001)$, or controlling for both $(b=1.30, p<.001)$. Similarly, information condition predicted desirability as a dating partner after controlling for change in physical attractiveness ratings $(b=2.40, p<.001)$. This leaves only mediational models that begin with information condition and end with change in attractiveness rating. The only model of that kind other than our predicted model (which was clearly consistent with full mediation) requires a three-step mediation from dating desirability to friendship desirability to change in physical attractiveness ratings. This model also clearly failed to meet the Step 4 criterion; that is, the cause to effect link remained strong and clearly significant when controlling for the mediator $(b=$ $.55, p<.001)$ one-sample $t$ test on the difference scores (Condition 1 minus Condition 2) on the effect measure. ${ }^{4}$ Step 2 tests for an effect of the cause on the mediator. In the within-subjects case, this is a $t$ test for the difference scores (between the two conditions) on the mediator variable. Step 3 tests for an effect of the mediator on the effect when controlling for the cause. In the within-subjects case, this is a regression in which the mediator's difference scores significantly predict the effect's difference scores.

Due to of the possibility of interactions, Judd et al. (2001) recommend that one also include in this regression, as an additional predictor, the sum scores for the two conditions for the mediator. If this sum term is significant, it qualifies the interpretation of results. We did this in all analyses reported and in no case was the sum term significant.

Finally, Step 4 tests whether the results are consistent with complete mediation by whether the cause-effect link drops to nonsignificance when the mediator is included as a predictor. In the within-subjects case, this means the intercept in the Step 3 regression analysis should not be significant. (The idea here is that the intercept in this analysis represents the deviation from 0 when predicting the effect due to factors other than the mediator. The only factor other than mediator that could account for a deviation from 0 would be the causal variable; thus, if this deviation differs from 0 , it is testing the effect of the cause over and above the mediator.)

Our model (see Figure 1) predicts the following two mediations that can be tested using the Judd et al. (2001) procedure: (a) rated friendship desirability mediates the effect of manipulated personality trait desirability on

4. Judd et al.'s (2001) procedures are based on a twocondition within-subjects design. Thus, all results reported were done for the desirable versus undesirable comparison. We also conducted parallel analyses for the desirable versus no information and the no information versus undesirable contrasts. In all these mediation analyses, results were in the expected direction and, with only one exception, all steps for the contrast yielded results (significant or not) consistent with complete mediation. The one exception was for the desirable versus no information contrast for the information-to-friendship-to-dating mediation. 
Table 2. Mediation step $t$ values

\begin{tabular}{lrrrr}
\hline \hline Mediation & Step 1 & Step 2 & Step 3 & Step 4 \\
\hline Info $\rightarrow$ Friend $\rightarrow$ Dating & $15.09^{* * *}$ & $16.91^{* * *}$ & $11.44^{* * *}$ & 1.29 \\
Info $\rightarrow$ Dating $\rightarrow$ PhysAtt & $9.74^{* * *}$ & $15.09^{* * *}$ & $4.64 * * *$ & 1.41 \\
\hline \hline
\end{tabular}

Note. These results are consistent with complete mediation in both mediation analyses. Step 1 in the standard Baron and Kenny (1986) mediation approach tests the influence of the initial cause on the effect; Step 2 tests whether there is an effect of the cause on the mediator; Step 3 tests whether there is an effect of the mediator on the effect after controlling for the cause; Step 4 tests whether the cause-effect link drops to nonsignificance when the mediator is included as a predictor. For complete mediation, the first three steps should be significant and the last step should not be significant. The particulars of the present analysis are based on adaptation of the Baron-Kenny approach for within-subject experiments spelled out by Judd et al. (2001). Info = manipulated desirability of trait information; Friend = rated friendship desirability; Dating = rated dating desirability; PhysAtt = change in rated physical attractiveness from pretest to posttest (see also Footnotes 3, 4, and 5).

$* * * p<.001$.

rated dating desirability and (b) rated dating desirability mediates the effect of manipulated personality trait desirability on pretest to posttest change in rated physical attractiveness rating. ${ }^{5}$ As shown in Table 2, results of all four steps supported complete mediation for both mediations predicted by the model.

\section{Discussion}

This research supports the hypothesis that judgments of physical attractiveness of an opposite-sex individual can change after one learns about the personality of that individual. Further, this effect exists for both women and men; for targets who were independently rated as attractive, neutral, or unattractive; and for increasing physical attractiveness judgments after receiving desirable trait information versus no information and for decreasing physical attractiveness ratings after receiving undesirable trait information versus no information. In

5. Judd et al.'s (2001) approach cannot be applied to test the friendship-to-dating-to-attractiveness mediation. This is because Judd et al.'s method is based on a two-condition dichotomous causal variable, which worked fine (taking two conditions at a time, per footnote 4) for testing mediations of the effect of two-condition personality information manipulation comparisons on other variables. Desirability as friend is a continuous variable. Thus, it cannot serve as the causal variable in testing mediations using Judd et al.'s approach. As noted in footnote 3, we were able to test the friendship-to-dating-to-attractiveness mediation using HLM; we did so, and results were unambiguously consistent with compete mediation, as predicted by the model shown in Figure 1. addition, we obtained a set of results that, in a variety of statistical tests, were strongly consistent with a model of the underlying mechanisms (shown in Figure 1) in which desirability of personality traits leads to greater desirability of the target as a friend, which then leads to greater desirability of the target as a dating partner, which then leads to the target being evaluated as more physically attractive. First (as shown in Figure 3), the effect of the personality manipulation was clearly greatest for friendship, next greatest for dating, and next greatest for change in attractiveness ratings (an order of effect sizes that, notably, was exactly the opposite of what would be expected from the temporal sequence in which participants made their ratings). Second, and more important, a series of mediation tests (using both standard methods and multilevel modeling) were entirely consistent with full mediations in the predicted pattern as shown in Figure 1. As would be expected from the pattern of effect sizes, none of the tests of mediation for all possible alternative mediational models among these four variables met minimal conditions for even partial mediation (see footnote 3).

Past research has sometimes found an effect of personality information on judgments of general attractiveness, including as a dating partner (Keisling \& Gynther, 1993; Lampel \& Anderson, 1968). There are also previous studies showing information about the person can affect judgments of physical attractiveness when both the information and the 
target's physical appearance are presented simultaneously (Albada et al., 2002; Gross \& Crofton, 1977; Hassebrauck, 1986; Paunonen, 2006). Both of these groups of previous studies were limited for the present purposes in a variety of ways. In terms of methods, the most important issue is that the previous studies presented the two kinds of information simultaneously.

This design feature is problematic for determining how judgments of attractiveness may change over time. To obtain this information, the present study first presented an image of the person with no other information (and had participants rate the person's physical appearance). Only later, and after both a mentally demanding distraction task and rating many other photos, did participants again rate the physical appearance of the individual. Thus, our study represents the first test of whether personal information can actually change ratings of physical appearance after they were previously evaluated. This result is important because it demonstrates the substantial power of personality information, in that it is sufficient to overcome initial evaluations. In addition, this result may be of significance to social and cognitive psychologists outside the relationship domain in that it is possibly relevant to the issues of whether top-down cognitive processes can influence perceptual processes (Eberhardt et al., 2003; von Hippel et al., 1995).

Our analyses concerning the influence of gender yielded a small but significant interaction of information condition with gender. The direction of the effect was such that females placed a greater emphasis on trait information when rating attractiveness of males, than did males when rating females. This pattern is consistent with a large body of literature based in evolutionary theory that suggests men value physical features more when choosing a mate, while females place more value on personality variables such as status (Buss, 1989). On the other hand, the clear finding of a strong main effect for each gender is consistent with personality being of great importance to both genders (e.g., Li et al., 2002).

It is also possible that a participant's level of commitment to his or her own relationship could change the influence of information on personality. Using relationship status as a proxy for commitment, we tested this possibility and found no sign of any interaction of relationship status with any of our effects. This suggests the influence of information on judgments of attractiveness is similar regardless of commitment level.

There is also the possibility that the question order in the posttest contributed to the findings. The chosen order was opposite of the effect we predicted and done to minimize recency effects that could have artificially increased our effect. Thus, by testing all participants in this reverse order, we are both working against our hypothesis, while providing maximum power for testing the hypothesized effect under the most rigorous conditions.

An important contribution of the present study is its positing, and successful testing, of a systematic model of how reevaluation of physical appearance might take place. Specifically, we hypothesized that knowing a person has desirable personality traits leads to considering the person to be desirable as a friend, that desirability as a friend relates to desirability as a dating partner, and that desirability as a dating partner leads to seeing the person as more attractive. We predicted this last link because perceiving a person to be a desirable dating partner should lead one to expect, as well as motivate one to believe, that the person is physically attractive. Both the expectation and motivational aspects are consistent with the general findings in social cognition research, though in few cases they have been examined in the relationship domain of with regard to evaluations of physical attractiveness. Further, in the specifically romantic relationship context, this result may be related to the extensive work on idealization by Murray and colleagues (e.g., Murray \& Holmes, 1997; Murray, Holmes, \& Griffin, 1996).

The present study, of course, had both methodological strengths and weaknesses. Strengths include the use of both male and female participants with opposite-gendered targets. This is advantageous (from the point of view of sorting out opposite-sex romantic attraction) in relation to the most relevant previous studies (Gross \& Crofton, 1977; Hassebrauck, 1986; Paunonen, 2006), which used participants of only one gender or had 
participants rate targets of the same gender. As is typical in research on attraction (including most previous studies), we did not ask participants about their sexual orientation (which is often considered invasive). Based on previous studies in our subject pool, we expected that the percentage of nonheterosexuals in our sample would be small. Moreover, the presence of nonheterosexuals should only serve to work against our effect. We acknowledge that it is possible that the numbers happened to be higher in our particular sample and that somehow this group could be driving the results on the basis of some other kinds of mechanisms we have not considered. A second, more technical, methodological strength compared to previous work was the use of more standardized presentation procedures for the stimuli, including counterbalancing and placing targets in a standard circle so that only the head was visible.

A limitation of our design was its inability to address the persistence of the effect over time. It is possible that effects are transient. On the other hand, one might expect the role of personal information to become increasingly, rather than decreasingly, important as one both learns more such information and becomes increasingly interdependent with the other. Another issue is that our experiment demonstrated a causal effect of information on friendship desirability, dating desirability, and physical attractiveness. Aspects of the mediational model (the hypothesized effects of friendship desirability on dating desirability and of dating desirability on physical attractiveness) could be tested only by their consistency with a causal model. The consistency with the model was very strong, we tested several alternative models and found they were not a good fit to the data (see footnote 3), and such an approach to testing mediating mechanisms is standard in the social sciences. Nevertheless, further research testing each of the causal links experimentally would be valuable. Another issue is that our methods could not sort out the extent to which ratings of physical attractiveness correspond to changes in the actual perception of physical features of the target compared to changes in the evaluation of the appearance of the target or its features.
Both interpretations are consistent with our results, and we would strongly encourage future research focusing on this issue.

Regarding generalizability, a potential limitation was our use of all Caucasian target stimuli with a relatively mixed ethnicity sample, drawn entirely from a U.S. population. In fact, the previous literature suggests increased attraction for those of similar backgrounds (Watson et al., 2004). Thus, the use of a mixed-ethnicity sample that rated targets with dissimilar backgrounds may have worked against our effect. It is also possible that our pattern of results might be different in other cultural contexts, particularly where arranged marriage is the norm and people may have personal information prior to knowing what the person looks like. On the other hand, we might speculate that there would be even stronger effects of personal information on physical appearance judgments when people feel they have no choice about their romantic partner or that their partner was presumably well chosen for them by respected elders. Further, the use of a convenience sample of introductory psychology students from one university limits the generalizability of the findings. We expect that positive information would generally lead to increases in perceived attractiveness. The type of information that is viewed positively could vary across populations. For example, it is possible that those taking psychology courses value personality more highly than others. It is also possible that non-college-age participants or those who do not attend college would give relatively more or less value to personality or physical appearance. Still, it would seem unlikely that any such different valuing would reverse the pattern of effects observed in the present sample.

Finally, the most serious external validity limitation of the present work, as with most laboratory experiments, is with regard to generalizing to the real world. We believe the present study provides an experimental examination with high levels of control that increases our understanding of possible processes operating in perceived attractiveness. At the same time, we think it is important to acknowledge that the artificial situation in this study does not replicate natural interactions and serves to 
highlight the importance of examining communication processes in more naturalistic settings. We of course hope that the present research will provide a strong foundation for future work in relationship and communication science that can address all these various issues and limitations, as well as furthering the theoretical analysis by examining additional intervening steps as well as potential moderators.

We would like to conclude with some comments on potential applied implications. The present research suggests that initial judgments of physical attractiveness of oppositesex persons can be influenced by nonphysical factors such as personality trait information. Thus, while it may still be important to be physically attractive, it is also important to convey a desirable personality. These findings are particularly encouraging as cosmetic surgery becomes increasingly common. Hopefully, this research provides a more positive alternative by reminding people that personality goes a long way toward determining your attractiveness; it can even change people's impressions of how good looking you are.

\section{References}

Albada, K., Knapp, M. L., \& Theune, K. E. (2002). Interaction appearance theory: Changing perceptions of physical attractiveness through social interaction. Communication Theory, 12, 8-40.

Altman, I., \& Taylor, D. A. (1973). Social penetration: The development of interpersonal relationships. New York: Holt, Rinehart, \& Winston.

Anderson, N. (1968). Likeableness ratings of personalitytrait words. Journal of Personality and Social Psychology, 9, 272-279.

Aron, A., Dutton, D. G., Aron, E. N., \& Iverson, A. (1989). Experiences of falling in love. Journal of Social and Personal Relationships, 6, 243-257.

Aron, A., Fisher, H., \& Strong, G. (2006). Love. In D. Perlman \& A. Vangelisti (Eds.), Handbook of personal relationships (pp. 595-614). New York: Cambridge University Press.

Baron, R. M., \& Kenny, D. A. (1986). The moderatormediator variable distinction in social psychological research: Conceptual, strategic, and statistical considerations. Journal of Personality and Social Psychology, 51, 1173-1182.

Bryk, A., \& Raudenbush, S. W. (1992). Hierarchical linear models for social and behavioral research: Applications and data analysis methods. Newbury Park, CA: Sage.

Buss, D. M. (1989). Sex difference in human mate preferences: Evolutionary hypotheses tested in 37 cultures. Behavioral and Brain Sciences, 12, 1-49.
Buss, D. M., \& Barnes, M. (1986). Preferences in human mate selection. Journal of Personality and Social Psychology, 50, 559-570.

Buss, D. M., \& Kenrick, D. T. (1998). Evolutionary social psychology. In D. T. Gilbert, S. T. Fiske, \& G. Lindzey (Eds.), The handbook of social psychology (4th ed., Vol. 2, pp. 982-1026). New York: McGraw-Hill.

Cedrus. (1999). SuperLab Pro (2.0) [Computer software]. San Pedro, CA: Cedrus.

Dion, K., Berscheid, E., \& Walster, E. (1972). What is beautiful is good. Journal of Personality and Social Psychology, 24, 285-290.

Eagly, A. H., Ashmore, R. D., Makhijani, M. G., \& Longo, L. C. (1991). What is beautiful is good, but. . . : A meta-analytic review of research on the physical attractiveness stereotype. Psychological Bulletin, $110,109-128$.

Eberhardt, J. L., Dasgupta, N., \& Banaszynski, T. L. (2003). Believing is seeing: The effects of racial labels and implicit beliefs on facial perception. Personality and Social Psychology Bulletin, 29, 360-370.

Feingold, A. (1992). Good-looking people are not what we think. Psychological Bulletin, 111, 304-341.

Fisher, H. E. (1998). Lust, attraction, and attachment in mammalian reproduction. Human Nature, 9, 23-52.

Gross, A. E., \& Crofton, C. (1977). What is good is beautiful. Sociometry, 40, 85-90.

Hassebrauck, M. (1986). Perception of physical attractiveness influenced by similarity of attitudes. Perceptual and Motor Skills, 63, 185-186.

Hassin, R., \& Trope, Y. (2000). Facing faces: Studies on the cognitive aspects of physiognomy. Journal of Personality and Social Psychology, 78, 837-852.

Hendricks, S. S., \& Hendricks, C. (1993). Lovers as friends. Journal of Social and Personal Relationships, 2, 55-65.

Jensen-Campbell, L. A., Graziano, W. G., \& West, S. G. (1995). Dominance, prosocial orientation, and female preferences: Do nice guys really finish last? Journal of Personality and Social Psychology, 68, 427-440.

Judd, C. M., Kenny, D. A., \& McClelland, G. H. (2001). Estimating and testing mediation and moderation in within-subject designs. Psychological Methods, 6, 115-134.

Keisling, B. L., \& Gynther, M. D. (1993). Male perceptions of female attractiveness: The effects of targets' personal attributes and subjects' degree of masculinity. Journal of Clinical Psychology, 49, 190-195.

Kirby, D. M., \& Gardner, R. C. (1972). Ethnic stereotypes: Norms on 208 words typically used in their assessment. Canadian Journal of Psychology, 26, 140-154.

Kleck, R. E., \& Rubenstein, C. (1975). Physical attractiveness, perceived attitude similarity, and interpersonal attraction in an opposite-sex encounter. Journal of Personality and Social Psychology, 31, 107-114.

Kniffin, K. M., \& Wilson, D. S. (2004). The effect of nonphysical traits on the perception of physical attractiveness: Three naturalistic studies. Evolution and Human Behavior, 25, 88-101.

Lampel, A. K., \& Anderson, N. (1968). Combining visual and verbal information in an impression-formation task. Journal of Personality and Social Psychology, 9, $1-6$.

Langlois, J. H., Kalakanis, L., Rubenstein, A. J., Larson, A., Hallam, M., \& Smoot, M. (2000). Maxims or myths of beauty? A meta-analytic and theoretical review. Psychological Bulletin, 126, 390-423. 
Lauer, J., \& Lauer, R. (1985, June). Marriages made to last. Psychology Today, 22-26.

Levinger, G. (1994). Figure versus ground: Micro- and macroperspectives on the social psychology of personal relationships. In R. Erber \& R. Gilmour (Eds.), Theoretical frameworks for personal relationships (pp. 1-28). Hillsdale, NJ: Erlbaum.

Li, N., Bailey, J., \& Kenrick, D. T. (2002). The necessities and luxuries of mate preferences: Testing the tradeoffs. Journal of Personality and Social Psychology, 82, 947-955.

Moreland, R. L., \& Beach, S. R. (1992). Exposure effects in the classroom: The development of affinity among students. Journal of Experimental Social Psychology, $28,255-276$

Murray, S. L., \& Holmes, J. G. (1997). A leap of faith? Positive illusions in romantic relationships. Personality and Social Psychology Bulletin, 23, 586-604.

Murray, S. L., Holmes, J. G., \& Griffin, D. W. (1996). The self-fulfilling nature of positive illusions in romantic relationships: Love is not blind, but prescient. Journal of Personality and Social Psychology, 71, 1155-1180.

Nisbett, R. E., \& Wilson, T. D. (1977). The halo effect: Evidence for unconscious alteration of judgments. Journal of Personality and Social Psychology, 35, 250-256.
Paunonen, S. V. (2006). You are honest, therefore I like you and find you attractive. Journal of Research in Personality, 40, 237-249.

Perrett, D. I., Burt, M. D., \& Penton-Voak, I. S. (1999). Symmetry and human facial attractiveness. Evolution and Human Behavior, 20, 295-307.

Stapel, D. A., \& Koomen, W. (1997). Social categorization and perceptual judgment of size: When perception is social. Journal of Personality and Social Psychology, 73, 1177-1190.

Veenvliet, S. G., \& Paunonen, S. V. (2005). Person perception based on rape-victim testimony. Deviant Behavior, 26, 209-227.

von Hippel, W., Sekaquaptewa, D., \& Vargas, P. (1995). On the role of encoding processes in stereotype maintenance. In M. Zanna (Ed.), Advances in experimental social psychology (pp. 177-254). San Diego, CA: Academic Press.

Watson, D., Klohnen, E. C., Casillas, A., Nus Simms, E., Haig, J., \& Berry, D. (2004). Match makers and deal breakers: Analyses of assortative mating in newlywed couples. Journal of Personality, 72, 1029-1068.

Wilkinson, L. (1999). Statistical methods in psychology journals: Guidelines and explanations. American Psychologist, 54, 594-604.

Wilson, P. R. (1968). Perceptual distortion of height as a function of ascribed academic status. Journal of Social Psychology, 74, 97-102. 